\title{
IDENTIFICATION OF AN INTESTINAL MICROBIOTA SIGNATURE ASSOCIATED WITH HOSPITALIZED PATIENTS WITH DIARRHEA
}

\author{
Nima MohammadzadeH ${ }^{1}$, Behrooz SAdeghi Kalani ${ }^{1}$, Shahin BolORI ${ }^{2}$, \\ AZADEH AZADEGAN ${ }^{3}$, AFSANEH GHOLAMI ${ }^{4}$, ROKHSAREH MOHAMMADZADEH ${ }^{1}$ \\ and FARAMARZ MASJEDIAN JAZI ${ }^{1} *$ \\ ${ }^{1}$ Department of Microbiology, School of Medicine, Iran University of Medical Sciences, \\ Tehran, Iran \\ ${ }^{2}$ Department of Microbiology, Faculty of Medicine, Shahid Beheshti University of \\ Medical Sciences, Tehran, Iran \\ ${ }^{3}$ Department of Microbiology, School of Medicine, Tehran University of Medical \\ Sciences, Tehran, Iran \\ ${ }^{4}$ Department of Microbiology, Karaj Branch, Islamic Azad University, Karaj, Iran
}

(Received: 11 July 2018; accepted: 22 September 2018)

\begin{abstract}
As an important global health challenge, diarrhea kills nearly two million people each year. Postinfectious irritable bowel syndrome (IBS) usually manifests itself as the diarrhea-predominant subtype. Small intestinal bacterial overgrowth has been observed more frequently in patients with IBS compared to healthy controls. However, the pathophysiology of IBS is not fully understood, and based on recent evidences, altered gut microbiota is involved in the pathogenesis of IBS. Therefore, we aimed to compare the microbiome in hospitalized patients with diarrhea and healthy individuals. Thirty patients and 10 healthy controls were included into this casecontrol study. Microbial count was performed using quantitative real-time polymerase chain reaction method using bacterial $16 \mathrm{~S}$ rRNA gene. Clostridium cluster IV and Bacteroides were significantly more frequent in the patients compared with the healthy individuals ( $p=0.02$ and 0.023 , respectively). However, the quantity of Enterococcus and Bifidobacterium groups were significantly higher in healthy controls than in diarrheal group ( $p=0.000076$ and 0.001 , respectively). The results showed that the number of bacteria in all bacterial groups was significantly different between healthy individuals and diabetic group, whereas the difference between the healthy group and IBS was not significant for Bifidobacterium group. The findings of this study outlined the relationship between diarrhea, IBS, and diabetes disease and bacterial composition. It could be concluded that modifying the bacterial composition by probiotics can be helpful in the control and management of the mentioned disease.
\end{abstract}

*Corresponding author; E-mail: fmasjedian@yahoo.com 
Keywords: diarrhea, irritable bowel syndrome, microbiota, diabetes

\section{Introduction}

Recent reports focused on the reality of a positive relationship between human health and the microbial flora that colonize the human gut. Among the gut bacteria, which behave as a functional human organ, probiotics represent the Holy Grail because they are related to a broad spectrum of positive effects on host health, including positive effects on host longevity [1]. As a functional gastrointestinal (GI) disorder, irritable bowel syndrome (IBS) is characterized by abdominal pain and changes in the pattern of bowel movements associated with bowel habit change such as diarrhea and constipation without any evidence of underlying damage $[2,3]$. Although IBS is not known as a serious disorder, $10 \%-15 \%$ of the adult population in the developed world has been observed affected with the disorder; however, the pooled prevalence of IBS varies considerably, both by geographical location and by applied diagnostic criteria [4-6]. It is more common in South America and less common in Southeast Asia and the high prevalence of IBS is joined by vast societal monetary burdens and negative impacts on the quality of life in the afflicted individuals with the disorder [7, 8]. IBS does not lead to dangerous conditions in most patients, but can be associated with increased side effects such as chronic pain and fatigue and reduced work productivity [9, 10]. Researchers have reported that the high incidence of IBS together with the associated comorbidities can increase social costs and can also greatly affect the patient's quality of life $[8,11]$.

Although the pathophysiology of IBS is not fully understood, several hypotheses have been proposed. Acute GI infections increase the risk of developing and expanding IBS. Prolonged fever, anxiety, and depression are the other factors that increase the likelihood of developing the syndrome. The biochemical signaling taken place between the GI tract and the central nervous system (the brain-gut axis) has been accepted as the major pathogenetic mechanism of IBS, which suggests that IBS occurs due to disturbances in the interoperability of the brain-intestinal axis and is correlated with a dysfunction of the GI autonomic nervous system [4]. Other theories including gut motility disorders, pain sensitivity, infections including small intestinal bacterial overgrowth, neurotransmitters, genetic factors, and food sensitivity have also known as the causes of the disorder [12].

Despite the considerable burden of IBS, treatment alternatives stay restricted and look into the etiology, and pathophysiology of this multifactorial disorder is continuous [13]. The growing evidences have indicated that IBS might present due to other potential mechanisms including gut microbiota and low-grade inflammation/immune activation [14, 15]. Comparisons of IBS patients with healthy 
participant in several lines of literatures have shown that the microbiota differs significantly between groups, which demonstrate the putative role of gut microbiota in IBS [16, 17]. Postinfectious IBS; colonic fecal microbiota transplantation; and therapeutic effects of probiotics, prebiotics, synbiotics, and non-systemic antimicrobials have also supported the contribution of the gut microbiota to the pathophysiology of IBS [18-23].

Given the evidence that approximately $10 \%$ of IBS patients' symptoms began following an episode of infectious diarrhea and based on our knowledge of the relationship between alteration of gut microbiota and inflammation of gut, we encouraged to compare the microbiome in hospitalized patients with diarrhea and healthy individuals.

\section{Materials and Methods}

\section{Participant and sampling}

Thirty affected hospitalized participants with diarrhea (age: $56 \pm 8$ years) who referred to Institute of Endocrinology and Metabolism Research and Training Center, Iran University of Medical Sciences in Tehran, Iran were recruited into the study as the case group. Twenty non-diarrheal individuals, matched for age, gender, and their current living environment were recruited as the healthy participants. Stool samples from the patients and healthy individuals were collected. Sterile cups were used to instant stool sampling after defecation and brought to the laboratory within $2 \mathrm{~h}$. Collected stool samples were instantly stored in microbiology laboratory at $-70{ }^{\circ} \mathrm{C}$ upon arrival.

Pregnancy, lactation, organic GI disease, severe systematic disease, major or complicated abdominal surgery, severe endometriosis, and dementia were the exclusion criteria for patients, and intestinal disturbances (including lactose intolerance and celiac disease), ongoing antibiotic treatment, and all exclusion criteria of the patients were considered as the exclusion criteria for controls.

This study was approved by research ethics committees of Iran University of Medical Sciences and according to Declaration of Helsinki. A signed informed consent form was received from each of the participant and they were ensured anonymity of all information.

\section{DNA extraction}

An amount of $200 \mathrm{mg}$ of each fecal sample was used for bacterial total DNA extraction. Extraction of total DNA from all stool specimens was performed using 
QIAamp $^{\circledR}$ DNA Stool Mini Kit (Qiagen Retsch GmbH, Hannover, Germany) according to the manufacturer's instruction. Quality and quantity of the extracted DNA was measured by Nanodrop spectrophotometer (Nanodrop Technologies, Wilmington, DE, USA) and the DNA integrity was assessed by $1 \%$ agarose gel-electrophoresis. Finally, entire extracted DNA samples were immediately transferred into $-20{ }^{\circ} \mathrm{C}$ storage.

Microbial quantification by real-time polymerase chain reaction (PCR)

Briefly, all bacterial 16S rRNA sequences were extracted from SILVA High Quality Ribosomal RNA database [30], then converted into 16S rDNA, and specific probe and primer sequences were designed in several steps using various databases including NCBI, probebase, IDT and EMBL-EBI, and also AlleleID software (version 7.5, Humana Press, USA). Characteristics of primers and TaqMan probes are demonstrated in Table I.

Using 16S rDNA gene-specific primers and probes, amplification of target $16 \mathrm{~s}$ rDNA was performed in Rotor-Gene 6000 real-time PCR cycler (Qiagen Corbett, Hilden, Germany) by real-time TaqMan quantitative PCR (qPCR). Each reaction mixture in a total volume of $20 \mu \mathrm{l}$, which contained $0.5 \mu \mathrm{l}$ of forward primer, $0.5 \mu \mathrm{l}$ of reverse primer, $0.5 \mu \mathrm{l}$ of TaqMan probe, $12 \mu \mathrm{l}$ of Probe Ex Taq (probe qPCR) Master Mix (Takara Bio, Shiga, Japan), $1 \mu 1$ of template DNA, and $5.5 \mu 1$ sterilized ultrapure water, was run to amplify the target region under following real-time qPCR cycling condition: an initial holding at $95^{\circ} \mathrm{C}$ for $30 \mathrm{~s}$, followed by 40 cycles of denaturation at $95{ }^{\circ} \mathrm{C}$ for $5 \mathrm{~s}$, and annealing/extension at $60^{\circ} \mathrm{C}$ for 30 s. No template reaction was used as the negative control. Bifidobacterium bifidum ATCC 29521, Lactobacillus acidophilus ATCC 4356, and Fusobacterium nucleatum ATCC 25586 were provided from the American Type Culture Collection (ATCC) and were used as the bacterial standard strains. All qPCR runs were carried out in triplicate, and averaged numbers were used for calculation and analysis.

\section{Bacterial count}

Determination of the number of Lactobacillus, Bifidobacterium, Fusobacterium, and Prevotella groups in each sample was performed after construction of standard curves based on 10-fold serial dilutions of bacterial standard strains genomic DNA of known concentration from pure cultures, corresponding to $10^{1}-10^{10}$ copies per gram feces. According to Applied Biosystems tutorials, standard curves were created and were normalized to the copy number of the $16 \mathrm{~S}$ rRNA gene for each species. If there was a copy number-unknown species of 


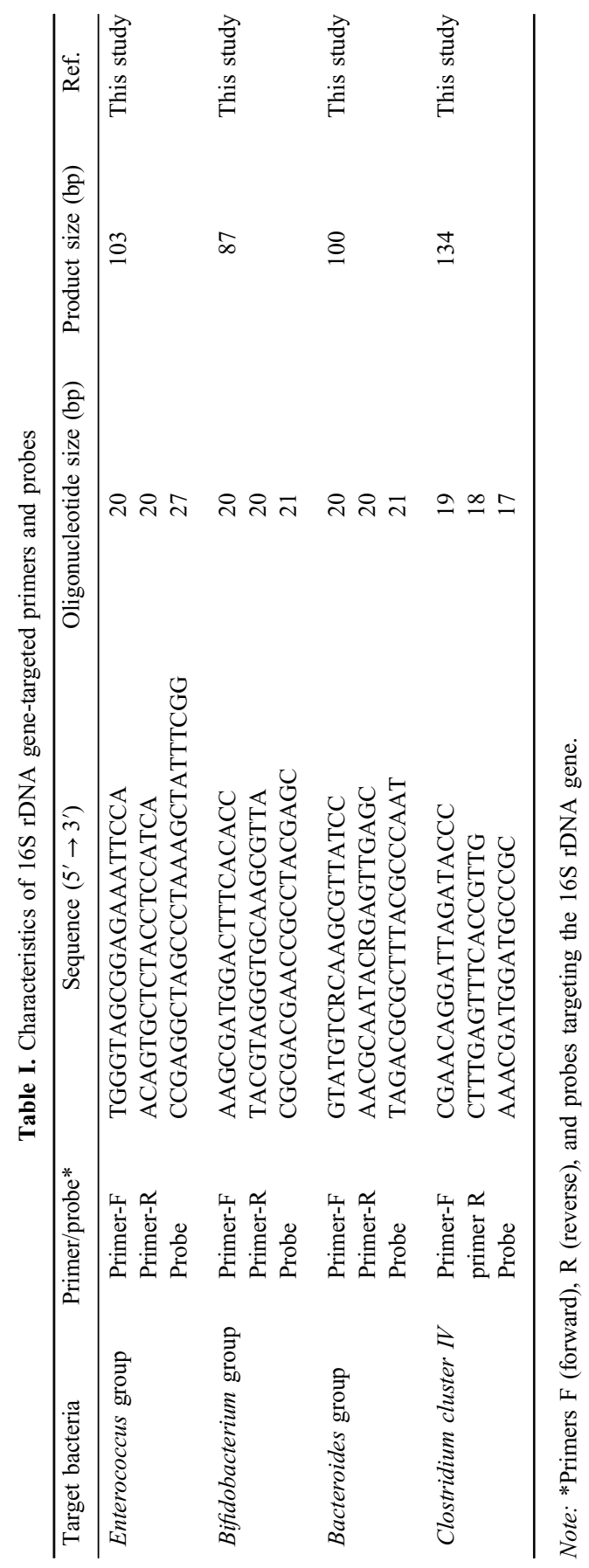


$16 \mathrm{~S}$ rRNA operon, average operon numbers of the closest bacterial taxa obtained from the ribosomal RNA database rrnDB was used as the operon copy number. The threshold cycle values $(\mathrm{Ct})$ obtained from the standard curves were applied to determine bacterial copy number per gram stool.

\section{Sample size and statistical analysis}

SPSS version 20.0 software (SPSS Inc., Chicago, IL, USA) and Minitab version 16.2.0 (State College, PA, USA) were used for statistical analysis. According to a predicted difference of at least $2 \times 10^{5}$ copies per gram of feces in the mean bacterial numbers between the healthy individuals and patients, the sample size of 20 subjects provided the sufficient power $(80 \%)$, considering a type I error of 0.05 and effect size of 0.4176 . For group comparison, independent sample $t$-test was used and the Pearson's correlation was assessed for linear correlation analysis. A $p<0.05$ was considered statistically significant. All the descriptive data have been expressed as mean \pm standard deviation. The Kolmogorov-Smirnov test was applied to test for a normal distribution.

\section{Results}

\section{Participants}

Thirty participants with diarrhea with the mean age of $56 \pm 8$ years and 10 healthy individuals with the mean age of $51 \pm 4$ years were recruited to the study; 14 participants with diarrhea were males and 16 were females. Healthy individuals included 4 males and 7 females.

\section{qPCR analysis of bacterial groups}

This case-control study qPCR analysis was aimed to assess the differences in composition of fecal microbiota in patients with diarrhea and healthy participant for four groups of bacteria including Bacteroides, Clostridium cluster IV, Enterococcus, and Bifidobacterium groups. As indicated in Table II, the results showed that fecal microbiota in diarrheal cases and healthy individuals were significantly different for all the studied bacterial groups. Clostridium cluster $I V$ and Bacteroides were significantly more frequent in the patients compared with the healthy individuals ( $p=0.02$ and 0.023, respectively). However, the quantity of Enterococcus and Bifidobacterium groups was significantly higher in healthy controls than in diarrheal 


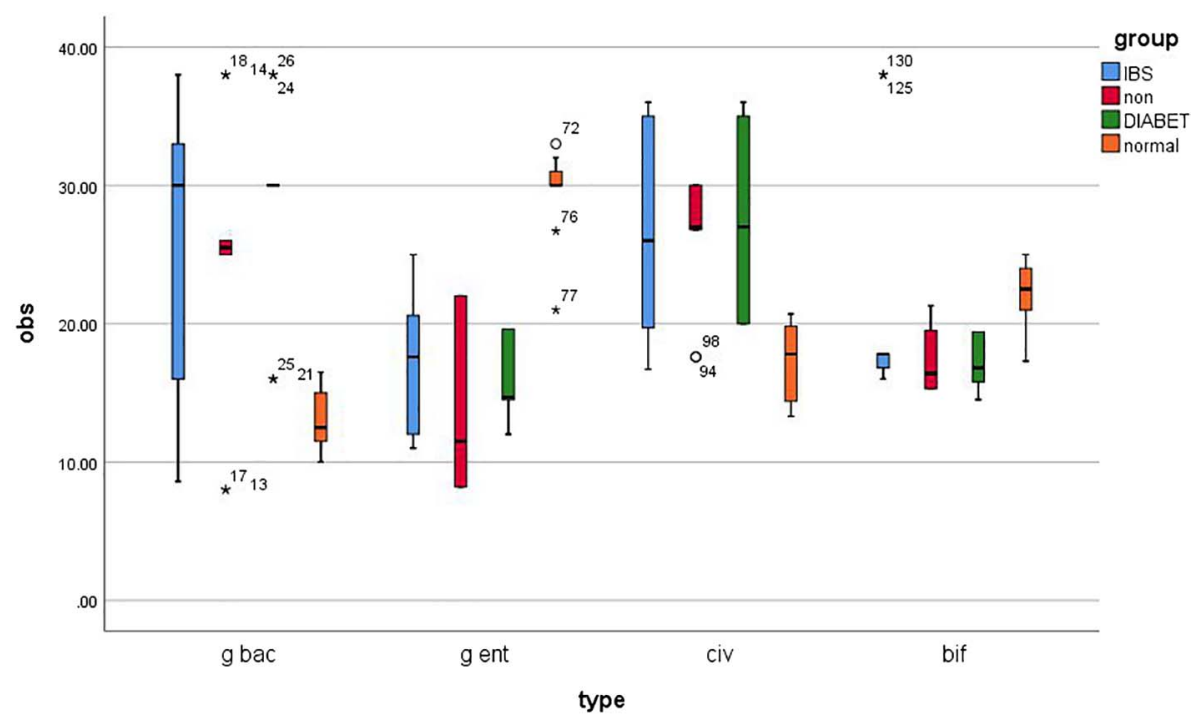

Figure 1. Bacterial groups quantified by real-time PCR and expressed as copy number of bacterial groups per gram stool in human adults with diarrhea (red boxes; $N=10$ ), IBS (blue boxes; $N=10$ ), diabetes (green boxes; $N=10$ ), and healthy controls (orange boxes; $N=10$ ). Boxes show the upper $(75 \%)$ and the lower $(25 \%)$ percentiles of the data. Whiskers indicate the highest and the smallest values. *Outlier points

group ( $p=0.000076$ and 0.001 , respectively). Differences of intestinal bacterial genera in the study groups were represented in Table II and Figure 1.

The correlations between concentration of the bacterial species in healthy-diabetic and healthy-IBS groups

Some of the participants in this study had background diseases of diabetes and IBS; therefore, the correlation or, in other words, the difference in bacterial groups between healthy individuals and those with any of the mentioned diseases was investigated. The results showed that the number of bacteria in all bacterial groups was significantly different between healthy individuals and diabetic group, whereas the difference between the healthy group and IBS was not significant for Bifidobacterium group. The mean quantity of Bacteroides, Clostridium cluster $\mathrm{IV}$, and Bifidobacterium groups was significantly higher in diabetic group than in nondiabetic individuals ( $p=0.000043, p=0.001$, and $p<0.001$, respectively). Bacteroides, Clostridium cluster IV, and Bifidobacterium were also significantly more frequent in the IBS patients compared with the healthy individuals $(p=0.035$, 0.006 , and 0.052 , respectively). Bacteroides concentration was higher both in 
diabetic group and IBS patients than in healthy group $(p=0.000011$ and 0.000042 , respectively). The correlations between concentration of the bacterial species in healthy-diarrheal, healthy-diabetic, and healthy-IBS groups have been indicated.

\section{Discussion}

Correlation between imbalance normal microbial community and GI conditions such as inflammatory bowel disease and IBS, and wider systemic manifestations of disease such as obesity, type 2 diabetes, and atopy have previously been reported in several reports $[6,13,14]$. Although intensive research over the past two decades has been carried to find the etiology of IBS, its pathophysiology remains poorly understood [19]. Diarrhea, constipation, or mixed type are the symptoms of IBS. Based on this knowledge, we hypothesized that intestinal microbiota composition in patients with diarrhea is different from healthy control participant. In this study, the number of four groups of bacteria including Bacteroides, Clostridium cluster IV, Enterococcus, and Bifidobacterium was measured using qPCR in patients and healthy participants. Our data showed that fecal microbiota in diarrheal cases and healthy individuals were significantly different for all the studied bacterial groups.

In comparison to healthy controls, our data showed that the copy number of Bacteroides and Clostridium cluster IV group was significantly higher in participants with diarrhea. In contrast with this study, Swidsinski et al. [24] using fluorescence in situ hybridization analysis (FISH) demonstrated that the fecal microbiota in idiopathic diarrhea was markedly different, marked by reduction in concentrations of habitual Eubacterium rectale, Bacteroides, and Faecalibacterium prausnitzii groups. Using FISH analysis, a study conducted on patients by Soko et al. also showed that a reduction of a major member of Firmicutes, $F$. prausnitzii, is associated with a higher risk of postoperative recurrence of ileal Crohn's disease (CD). They found that $F$. prausnitzii exhibits anti-inflammatory effects, partly due to secreted metabolites able to block NF- $\mathrm{kB}$ activation and IL-8 production; therefore, counterbalancing dysbiosis using $F$. prausnitzii as a probiotic can be a promising strategy in CD treatment [25].

In addition to the diarrhea, which is designated as the principal diagnosis, some patients also have underlying diseases of diabetes and IBS. Therefore, we also decided to evaluate the association of each of the studied bacterial group with the underlying diseases. In agreement with findings of Vrakas et al. [26], Maukonen et al. [27], and Rajilić-Stojanović et al. [28], our observation showed that the concentrations of Clostridium cluster IV and Fusobacterium groups were relatively higher in the IBS group compared to the healthy participant. 
Consistently, Kerckhoffs et al. [29] showed that bifidobacteria levels in both fecal and duodenal brush samples of IBS patients were significantly lower ( $6 \pm 0.6 \mathrm{vs}$. $19 \pm 2.5, p<0.001)$ compared to healthy participants, which indicate a role for microbiotic composition in IBS pathophysiology. They analyzed fecal samples for the composition of the total microbiota using FISH and analyzed both fecal and duodenal brush samples for the composition of bifidobacteria using qPCR. Similarly, Rajilić-Stojanović et al. [28] and Si et al. [30] showed meaningful lower concentration of Bifidobacterium in IBS patients compared to the controls. In this study, we found that the level of Enterococcus group was significantly lower in the patients with IBS compared to the healthy group. This observation was in contrast with the findings of Zhuang et al. [31].

In line with our results, the study conducted by Rajilić-Stojanović, the intestinal microbiota including a twofold increased ratio of Bacteroidetes $(p=0.0002)$, approximately 1.5 -fold increase in numbers of Clostridium spp. $(p=0.005)$; a twofold decrease in the number of Bacteroidetes $(p=0.0001)$; a 1.5-fold decrease in numbers of Bifidobacterium and Faecalibacterium spp. $(p=0.05)$, of IBS patients differed significantly $(p=0.0005)$ from that of controls [28]. Characterization of the fecal microbiota using high-throughput sequencing of the 16S rRNA gene showed a significant increase of Bacteroidetes and Proteobacteria in the IBS group compared to the healthy participants [32]. Other report also confirmed our data and demonstrated that the mucosa-associated microbiota in patients with IBS is significantly different from healthy controls with increases in bacteroides and clostridia and a reduction in bifidobacteria in patients with IBS-D [33]. However, global and deep molecular analysis of microbiota signatures in fecal samples from patients with IBS disclosed a twofold decrease in the number of Bacteroidetes [33]. In this study, the level of Bifidobacterium was lower in IBS patients than controls, but this difference was not statistically significant.

This study revealed that the level of Bacteroides, Bifidobacterium, and Clostridium cluster $I V$ in patients with underlying disease of diabetes was significantly higher compared with those in their healthy counterparts. Microbiome profile showed a high level of Enterococcus group in healthy participant than individuals with diabetes. On the contrary to this study, Remely et al. [34] and Sedighi et al. [6] found no significant differences in copy number of genus Bifidobacterium between the case and control groups; nonetheless, Murri et al. [35] reported a significant decrease in the number of Bifidobacterium in the children with diabetes. Consistent with our findings, Larsen et al. [36] found that the ratio of Bacteroidetes was increased in diabetes' cases; however, Lambeth et al. [37] did not observe differences in the abundances of phyla Bacteroidetes. On the contrary to this study, data reported by Remely et al. [34] and Larsen et al. [36] showed no significant difference in the level of Clostridium cluster IV 
between patients with diabetes and healthy individuals. It is previously reported that total cholesterol and low-density lipoprotein cholesterol reduction are associated with dairy products enriched with Enterococcus faecium in participant having a normal lipid profile and participant with medium to moderate hypercholesterolemia [38].

Although participants included in this study were matched for age, gender, race, living environment, and non-interventions of medications and foods, which may affect the outcomes (such as different antibiotics, prebiotics, and probiotics), our findings were not completely consistent with previous reports, and controversial results regarding the dysbiosis of the gut microbiota in hospitalized patients with diarrhea as well as contradictory findings on the relationship between various bacterial groups and IBS and diabetes were observed. This discrepancy might be due to heterogeneity in various factors such as genetic background, ethnicity, geographical location, environmental and occupational exposures, medical history, possible underlying diseases/disorders, lifestyle, and diet habits of participant across studies. Non-significance in some of our results may have been related to a relatively small sample size.

On the whole, the results of this study indicate that diarrhea and the underlying disorders of IBS and diabetes in humans are associated with compositional changes in intestinal microbiota; however, we cannot conclude about the causality of this dysbiosis. Further studies are suggested to determine if microbial imbalance causes these medical conditions or changes in microbiota profile are a reflection of the disease state. Overall, it could be concluded that modifying the bacterial composition by probiotics can be helpful in the control and management of the mentioned disease.

\section{Acknowledgements}

The authors are grateful for the support provided by Iran University of Medical Sciences (Tehran, Iran).

\section{Conflict of Interest}

The authors declare no conflict of interest.

\section{References}

1. Ayala, F. R., Bauman, C., Cogliati, S., Leñini, C., Bartolini, M., Grau, R.: Microbial flora, probiotics, Bacillus subtilis and the search for a long and healthy human longevity. Microb Cell 4, 133-136 (2017). 
2. Lee, B. J., Bak, Y.-T.: Irritable bowel syndrome, gut microbiota and probiotics. J Neurogastroenterol Motil 17, 252-266 (2011).

3. Farahani, N. N., Jazi, F. M., Nikmanesh, B., Asadolahi, P., Kalani, B. S., Amirmozafari, N.: Prevalence and antibiotic susceptibility patterns of Salmonella and Shigella species isolated from pediatric diarrhea in Tehran. Arch Pediat Inf Dis 6, e57328 (2018).

4. Ohman, L., Simren, M.: New insights into the pathogenesis and pathophysiology of irritable bowel syndrome. Dig Liver Dis 39, 201-215 (2007).

5. Lovell, R. M., Ford, A. C.: Global prevalence of and risk factors for irritable bowel syndrome: A meta-analysis. Clin Gastroenterol Hepatol 10, 712-721.e4 (2012).

6. Sedighi, M., Razavi, S., Navab-Moghadam, F., Khamseh, M. E., Alaei-Shahmiri, F., Mehrtash, A., Amirmozafari, N.: Comparison of gut microbiota in adult patients with type 2 diabetes and healthy individuals. Microb Pathog 111, 362-369 (2017).

7. Drossman, D. A., Camilleri, M., Mayer, E. A., Whitehead, W. E.: AGA technical review on irritable bowel syndrome. Gastroenterology 123, 2108-2131 (2002).

8. Chey, W. D., Kurlander, J., Eswaran, S.: Irritable bowel syndrome: A clinical review. JAMA 313, 949-958 (2015).

9. Simrén, M., Axelsson, J., Gillberg, R., Abrahamsson, H., Svedlund, J., Björnsson, E. S.: Quality of life in inflammatory bowel disease in remission: The impact of IBS-like symptoms and associated psychological factors. Am J Gastroenterol 97, 389-396 (2002).

10. Bercik, P., Verdu, E. F., Collins, S. M.: Is irritable bowel syndrome a low-grade inflammatory bowel disease? Gastroenterol Clin North Am 34, 235-245 (2005).

11. Simrén, M., Svedlund, J., Posserud, I., Björnsson, E. S., Abrahamsson, H.: Health-related quality of life in patients attending a gastroenterology outpatient clinic: Functional disorders versus organic diseases. Clin Gastroenterol Hepatol 4, 187-195 (2006).

12. Ohman, L., Simrén, M.: Pathogenesis of IBS: Role of inflammation, immunity and neuroimmune interactions. Nat Rev Gastroenterol Hepatol 7, 163-173 (2010).

13. Dupont, H.: Review article: Evidence for the role of gut microbiota in irritable bowel syndrome and its potential influence on therapeutic targets. Aliment Pharmacol Ther 39, 1033-1042 (2014).

14. Ghoshal, U. C., Kumar, S., Mehrotra, M., Lakshmi, C., Misra, A.: Frequency of small intestinal bacterial overgrowth in patients with irritable bowel syndrome and chronic nonspecific diarrhea. J Neurogastroenterol Motil 16, 40-46 (2010).

15. Karantanos, T., Markoutsaki, T., Gazouli, M., Anagnou, N. P., Karamanolis, D. G.: Current insights in to the pathophysiology of irritable bowel syndrome. Gut Pathog 2, 3 (2010).

16. Kassinen, A., Krogius-Kurikka, L., Mäkivuokko, H., Rinttilä, T., Paulin, L., Corander, J., Malinen, E., Apajalahti, J., Palva, A.: The fecal microbiota of irritable bowel syndrome patients differs significantly from that of healthy subjects. Gastroenterology 133, 24-33 (2007).

17. Posserud, I., Stotzer, P.-O., Björnsson, E. S., Abrahamsson, H., Simrén, M.: Small intestinal bacterial overgrowth in patients with irritable bowel syndrome. Gut 56, 802-808 (2007).

18. Carroll, I. M., Ringel-Kulka, T., Keku, T. O., Chang, Y.-H., Packey, C. D., Sartor, R. B., Ringel, Y.: Molecular analysis of the luminal-and mucosal-associated intestinal microbiota in diarrhea-predominant irritable bowel syndrome. Am J Physiol Gastrointest Liver Physiol 301, G799-G807 (2011).

19. Carroll, I. M., Ringel-Kulka, T., Siddle, J. P., Ringel, Y.: Alterations in composition and diversity of the intestinal microbiota in patients with diarrhea-predominant irritable bowel syndrome. Neurogastroenterol Motil 24, 521-530 (2012). 
20. Halvorson, H. A., Schlett, C. D., Riddle, M. S.: Postinfectious irritable bowel syndrome A meta-analysis. Am J Gastroenterol 101, 1894-1899 (2006).

21. Ortiz-Lucas, M., Tobias, A., Saz, P., Sebastián, J. J.: Effect of probiotic species on irritable bowel syndrome symptoms: A bring up to date meta-analysis. Rev Esp Enferm Dig 105, 19-36 (2013).

22. Behrooz, S. K., Lida, L., Ali, S., Mehdi, M., Rasoul, M., Elnaz, O., Farid, B. T., Gholamreza, I.: Study of MazEF, sam, and phd-doc putative toxin-antitoxin systems in Staphylococcus epidermidis. Acta Microbiol Immunol Hung 65, 81-91 (2018).

23. Kalani, B. S., Irajian, G., Lotfollahi, L., Abdollahzadeh, E., Razavi, S.: Putative type II toxin-antitoxin systems in Listeria monocytogenes isolated from clinical, food, and animal samples in Iran. Microb Pathog 122, 19-24 (2018).

24. Swidsinski, A., Loening-Baucke, V., Verstraelen, H., Osowska, S., Doerffel, Y.: Biostructure of fecal microbiota in healthy subjects and patients with chronic idiopathic diarrhea. Gastroenterology 135, 568-579 (2008).

25. Sokol, H., Pigneur, B., Watterlot, L., Lakhdari, O., Bermúdez-Humarán, L. G., Gratadoux, J. J., Blugeon, S., Bridonneau, C., Furet, J. P., Corthier, G., Grangette, C., Vasquez, N., Pochart, P., Trugnan, G., Thomas, G., Blottière, H. M., Doré, J., Marteau, P., Seksik, P., Langella, P.: Faecalibacterium prausnitzii is an anti-inflammatory commensal bacterium identified by gut microbiota analysis of Crohn disease patients. Proc Natl Acad Sci U S A 105, 16731-16736 (2008).

26. Vrakas, S., Mountzouris, K. C., Michalopoulos, G., Karamanolis, G., Papatheodoridis, G., Tzathas, C., Gazouli, M.: Intestinal bacteria composition and translocation of bacteria in inflammatory bowel disease. PLoS One 12, e0170034 (2017).

27. Maukonen, J., Satokari, R., Mättö, J., Söderlund, H., Mattila-Sandholm, T., Saarela, M.: Prevalence and temporal stability of selected clostridial groups in irritable bowel syndrome in relation to predominant faecal bacteria. J Med Microbiol 55, 625-633 (2006).

28. Rajilić-Stojanović, M., Biagi, E., Heilig, H. G., Kajander, K., Kekkonen, R. A., Tims, S., de Vos, W. M.: Global and deep molecular analysis of microbiota signatures in fecal samples from patients with irritable bowel syndrome. Gastroenterology 141, 1792-1801 (2011).

29. Kerckhoffs, A. P., Samsom, M., van der Rest, M. E., de Vogel, J., Knol, J., Ben-Amor, K., Akkermans, L. M.: Lower Bifidobacteria counts in both duodenal mucosa-associated and fecal microbiota in irritable bowel syndrome patients. World J Gastroenterol 15, 2887-2892 (2009).

30. Si, J.-M., Yu, Y.-C., Fan, Y.-J., Chen, S.-J.: Intestinal microecology and quality of life in irritable bowel syndrome patients. World J Gastroenterol 10, 1802-1805 (2004).

31. Zhuang, X., Xiong, L., Li, L., Li, M., Chen, M.: Alterations of gut microbiota in patients with irritable bowel syndrome: A systematic review and meta-analysis. J Gastroenterol Hepatol 32, 28-38 (2017).

32. Carroll, I. M., Ringel-Kulka, T., Siddle, J. P., Klaenhammer, T. R., Ringel, Y.: Characterization of the fecal microbiota using high-throughput sequencing reveals a stable microbial community during storage. PLoS One 7, e46953 (2012).

33. Parkes, G., Rayment, N. B., Hudspith, B. N., Petrovska, L., Lomer, M. C., Brostoff, J., Whelan, K., Sanderson, J. D.: Distinct microbial populations exist in the mucosa-associated microbiota of sub-groups of irritable bowel syndrome. Neurogastroenterol Motil 24, 31-39 (2012). 
34. Remely, M., Dworzak, S., Hippe, B., Zwielehner, J., Aumüller, E., Brath, H., Haslberger, A.: Abundance and diversity of microbiota in type 2 diabetes and obesity. J Diabetes Metab 4, 2 (2013).

35. Murri, M., Leiva, I., Gomez-Zumaquero, J. M., Tinahones, F. J., Cardona, F., Soriguer, F., Queipo-Ortuño, M. I.: Gut microbiota in children with type 1 diabetes differs from that in healthy children: A case-control study. BMC Med 11, 46 (2013).

36. Larsen, N., Vogensen, F. K., van den Berg, F. W., Nielsen, D. S., Andreasen, A. S., Pedersen, B. K., Al-Soud, W. A., Sørensen, S. J., Hansen, L. H., Jakobsen, M.: Gut microbiota in human adults with type 2 diabetes differs from non-diabetic adults. PLoS One 5, e9085 (2010).

37. Lambeth, S. M., Carson, T., Lowe, J., Ramaraj, T., Leff, J. W., Luo, L., Bell, C. J., Shah, V. O.: Composition, diversity and abundance of gut microbiome in prediabetes and type 2 diabetes. J Diabetes Obes 2, 1-7 (2015).

38. Rebolledo, C., Cuevas, A., Zambrano, T., Acuña, J. J., Jorquera, M. A., Saavedra, K., Martínez, C., Lanas, F., Serón, P., Salazar, L. A., Saavedra, N.: Bacterial community profile of the gut microbiota differs between hypercholesterolemic subjects and controls. BioMed Res Int 2017, 8127814 (2017). 